\title{
Development of a Web-based learning tool for undergraduate health professionals studying applied anatomy
}

\author{
Valerie Cooper* and Mike McConnell** \\ * School of Health Sciences, The Robert Gordon University \\ ** Centre for Learning and Assessment, The Robert Gordon University \\ email:v.cooper@rgu.ac.uk
}

An understanding of anatomy is an essential component of degree courses for health professionals. A key learning outcome for physiotherapy students is to be able to observe, analyse and explain the sequence of normal anatomical movement patterns for the upper and lower limb. However, when there is a requirement for such learning to be practicebased it is not always possible to provide students with the necessary resources for independent study. This paper describes the development and evaluation of a Web-based tool that allows students to practise and test their human movement analysis skills independently, at their own pace. The tool utilizes video clips of common muscle movements and multiple-choice questions with dynamic feedback to promote understanding. The aims of the tool and its evaluation by a sample group of forty-five students is described. Suggestions for future development are discussed.

\section{Introduction}

It is a commonly accepted maxim of education that independent learning and directed study accommodate individual learning styles and allow students to acquire basic facts and principles. Such practices are increasingly desirable in the current educational climate as they allow teaching staff to use valuable contact time to address higher cognitive skills. However, when there is a requirement for such learning to be practice-based it is not always possible to provide students with the necessary resources for independent study.

In teaching anatomy to health professionals, such as physiotherapists, it is desirable to facilitate their understanding of function, rather than merely the ability to identify structure. Carr, Shepherd and Ada (1995) stress the importance of a clear understanding of 
normal functional movement. The ability to assess patient problems and develop a treatment strategy is dependent on comparing adaptive dysfunctional movement patterns to the functional norm. The skill of normal movement analysis is thus a fundamental requirement for physiotherapists in all clinical areas, as pathokinesiology is one of its main underpinning theories (Rothstein, 1986). Watson (1999) suggests that breaking movements into their component subsystems enables students to develop the skills to analyse problems.

The application of movement analysis is therefore an obvious requirement in physiotherapy, but other health professions also make use of these techniques. WilliamsPedretti and Zoltan (1990) recommend that occupational therapists observe performance (with particular attention to motor and sensory integrative components) to evaluate physical dysfunction. Kielhofner (1997) suggests that occupational dysfunction is multidimensional, but recognizes physical and motor capacity as factors which should be considered by occupational therapists. Meanwhile Snell (1995) notes that medical students need to understand the functional organization of anatomical structures such as muscles and joints and how they control activities of the body so that accurate diagnosis, and therefore effective treatment, is provided.

The desired learning outcome for this skill in the anatomy module (for first year BSc Honours undergraduate physiotherapists at the Robert Gordon University) complements these suggestions: the student must be able to observe, analyse and explain the sequence of normal anatomical movement patterns for the upper and lower limbs and trunk.

Students are currently taught basic anatomical facts through keynote lectures, demonstrations on cadavers and self-study CD-ROMs and workbooks. They are encouraged to understand and apply this knowledge through tutorials and practical classes. In movement analysis classes, the students model for each other and demonstrate various functional movements such as stepping, standing from sitting, pushing and throwing. The 'model' student performs each movement repeatedly and the students observe, describe and explain the movements occurring at each joint and how they are produced. It is imperative that the students analyse exactly what they see; as each individual moves differently, there is no textbook 'right' answer. As there are many movements and many variations, practice with different models is essential.

The practical class setting traditionally provides these opportunities and allows the tutor to give feedback, but it is also necessary for students to practise outside classes. French, Neville and Laing (1994) stress the importance of practice between teaching sessions, but recognize that students may have to rely on internal or peer group feedback. An additional complicating factor is model tiredness, as this varies the movement with each repetition. Videos may help solve this problem and have thus been suggested as an adjunct to individual practice (French et al., 1994).

At the Robert Gordon University, students are currently assessed on their anatomy through a written examination and a practical viva, which includes both a live model and video footage. Prior to the examination, students may request tutorials on the components of the module they wish to revise; they invariably choose movement analysis. A Movement Analysis Tool was thus devised in order to support further and enhance independent study of this important skill by: 
- providing students with a model for practice;

- providing students with feedback on their attempts at movement analysis;

- supporting students' understanding of the process with additional information.

The tool is thus intended to engage the students' attention via interactive and visually stimulating elements such as MCQs, video clips, animations and graphics, but also to provide added value in the form of dynamic feedback and glossaries of muscles and movements.

\section{The Movement Analysis Tool}

The Movement Analysis Tool presents muscle movements for the upper and lower limbs, and is accessed via a Web browser. The tool is divided into sections, each detailing a particular muscle movement. Each section is prefaced by a video clip of a model demonstrating the movement. Video clips of the movement are available in two formats: animated GIFs, which are embedded in the Web pages and load automatically; or highquality MPEG clips which are accessible via hypertext links. Below the clips in each section, a series of multiple-choice/multiple-response questions is presented which tests students on their knowledge of the starting, intermediate and finishing positions, the muscles producing the movement and the type of muscle work. When students have chosen responses, they submit them by clicking a button. The server 'marks' the questions and provides individualized feedback related to the students' responses, including a personalized cumulative score. If students answer questions incorrectly, feedback includes a link to the relevant glossary, which permits them to find the correct muscles or identify attachments to discover where they went wrong.

HTML was used to create the Movement Analysis Tool. The online assessment application suite Question Mark Perception was also used. In addition to offering a variety of question types, Perception includes sophisticated and customizable reporting facilities which can detail individual or aggregate scores, allowing for instantaneous feedback and marking as mentioned, and tracking of student behaviour. As any HTML content may be combined with questions generated by the application, hypertext links and multimedia content are easily incorporated. It was thus easy to provide links to other pages such as the glossaries, as part of the feedback. HTML tags were also used to include the animations.

Animations for the Movement Analysis Tool were captured from the original videotape and converted to MPEG and AVI format using an MPEG encoder. AVI files were broken down into individual frames, converted to GIFs and reassembled as animations. Animated GIFs were used for two reasons: they provide a significant saving on file size compared with MPEG and, unlike MPEG, they can be embedded in the question pages. However, animated GIF files do not provide the same degree of resolution as MPEGs, nor can they be easily paused and restarted. It was thus decided to retain the option to display MPEGs in the finished tool, via separate hypertext links. This allows students to play the MPEGs, stop and start them as they wish, and resize them as necessary, using Microsoft's Media Player. As students access the tool over the University Intranet, file size was, in this instance, not deemed to be especially problematic. 
Figure 1: Students' attitudes to technology pre- and post-pilot

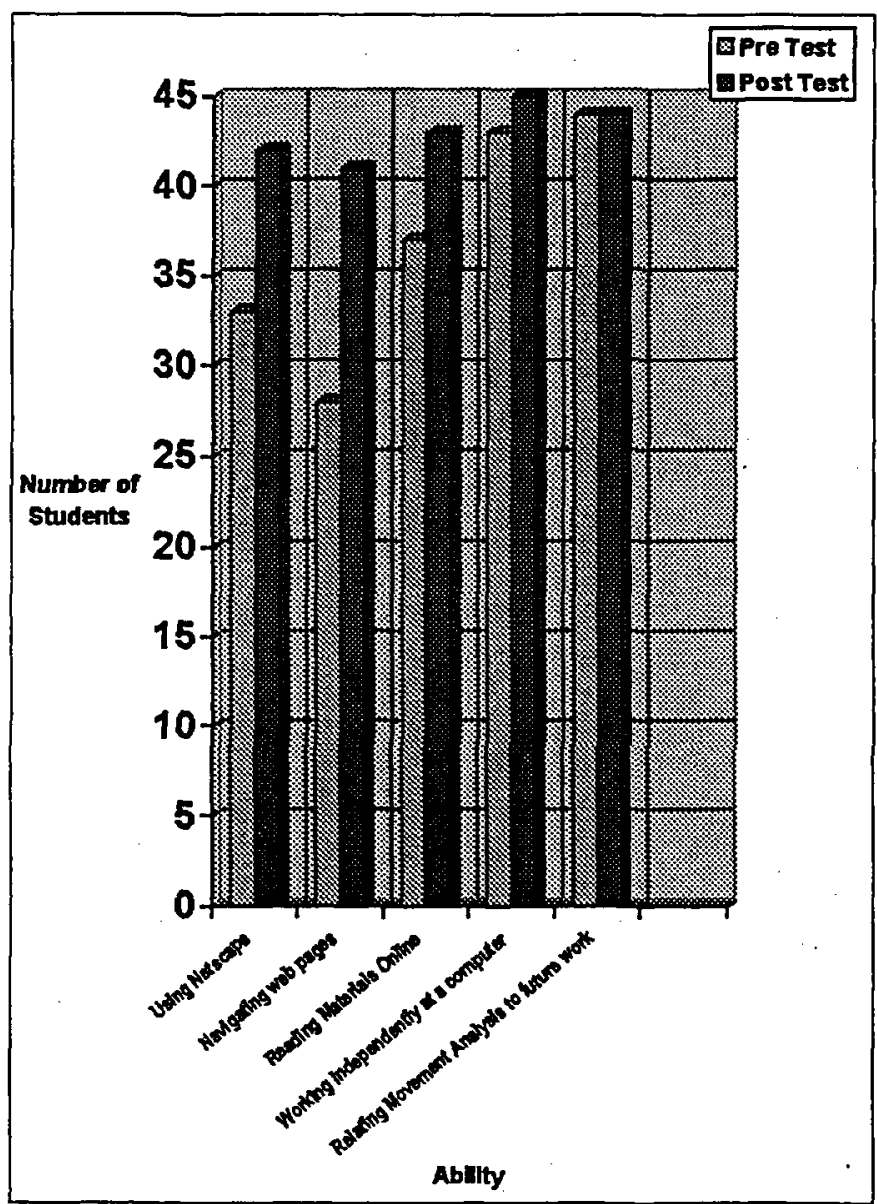

\section{Evaluation}

A pilot study of the tool was conducted using a sample of forty-five second-year physiotherapy students. Second-year students were selected as they were deemed to have the necessary anatomical knowledge to use the tool and would, moreover, understand the context for its use. The students were given an introductory session on how to use the tool and then divided into four groups. This ensured that they had adequate space and allowed technical and tutor support to be given as required. This reflected normal practice, as students would usually be given support from the tutor during their first session with a new learning tool, until they were confident enough to use the tool independently.

Brief pre- and post-session questionnaires were administered in order to assess students' levels of confidence about the subject matter, and their attitude towards technology. Attitudes about layout, navigation and content were assessed via Likert scales, but openended responses were also included for further details and to accommodate unanticipated reactions/responses. The pre-test questionnaire was administered during a brief introductory session. Students then spent thirty supervised minutes using the tool, before 


\begin{tabular}{lrrrrr}
\hline $\mathbf{n}=45$ & $\mathbf{1}$ & $\mathbf{2}$ & $\mathbf{3}$ & $\mathbf{4}$ & $\mathbf{5}$ \\
\hline Navigation & & & & & \\
Clear where you can go in Web pages $\mathrm{n}=43$ & 6 & 18 & 16 & 2 & 1 \\
Easy to get to where you want to go & 11 & 23 & 9 & 2 & 0 \\
Easy to bring up images and definitions & 18 & 18 & 7 & 1 & 0 \\
Easy to work out what is already completed $\mathrm{n}=44$ & 10 & 26 & 6 & 1 & 0 \\
& & & & & \\
Layout & 30 & 15 & 1 & 0 & 0 \\
Easy to read text & 18 & 17 & 7 & 2 & 3 \\
Easy to view graphics & 13 & 20 & 8 & 3 & 0 \\
Easy to locate other information & & & & & \\
& & & & & \\
Content & 32 & 12 & 2 & 0 & 0 \\
Style of writing is suitable & 24 & 15 & 6 & 0 & 0 \\
Complexity of content is suitable & 22 & 18 & 4 & 0 & 0 \\
Amount of content / length is suitable & & & &
\end{tabular}

$n=$ number of respondents

Figure 2: Students' attitudes to tool characteristics

completing the post-test questionnaire. Data were collected anonymously so that students would be candid in their comments about the materials.

Confidence levels in using IT for learning, and general knowledge of movement analysis before and after use of the tool are presented in Figure 1. Although the students surveyed were familiar with movement analysis from the first year of their course, many stated that they had forgotten elements over the summer, and needed to practise further. As can be seen from the chart, after the test an increase in student confidence had occurred, with thirty-one (69 per cent) students reporting an overall improvement in their confidence in the subject and the use of Web-based learning, eleven ( 24 per cent) reporting the same levels of confidence and only two students reporting a decrease in confidence. One of these latter students, however, attributed her decrease in confidence to an increased awareness of how much she had forgotten, so this was not considered a failing of the tool.

Figure 2 presents student attitudes towards characteristics of the tool (namely navigational aspects, layout and content), assessed via a Likert scale, where 1 indicates a well designed aspect and 5 indicates a poorly designed aspect. Very few negative attitudes were recorded, and some of the negative feedback related to graphics may be attributable to poor network performance during the course of one of the pilot sessions.

Students were then asked to comment on a series of attitudinal statements about the Web materials. Results are summarized in Figure 3, and again indicate generally positive attitudes towards the tool.

Students were asked to state the main advantages and disadvantages of using the Webbased materials for learning, and the three most commonly identified advantages and disadvantages are listed in Figure 4. It is encouraging to note that the biggest disadvantage was a generic problem with the network on the day and therefore not attributable to the tool

66 


\begin{tabular}{lccccc}
\hline & 1 & $\mathbf{2}$ & $\mathbf{3}$ & $\mathbf{4}$ & $\mathbf{5}$ \\
\hline Enjoyable to use & 7 & 26 & 10 & 2 & 1 \\
Helps you leam about the subject & 24 & 16 & 3 & 2 & 0 \\
Worth the time spent on it & 21 & 16 & 3 & 2 & 0 \\
Would help me to revise the subject & 26 & 14 & 2 & 3 & 0 \\
I would use it again in my own time & 12 & 26 & 3 & 1 & 3 \\
Will help me with future study in this discipline & 16 & 22 & 2 & 4 & 0 \\
\hline
\end{tabular}

I=strongly agree, $2=$ agree, $3=$ neutral, $4=$ disagree, $5=$ strongly disagree

Figure 3:Attitudinal statements about the tool

\begin{tabular}{ll}
\hline \multicolumn{1}{c}{ ADVANTAGES } & \multicolumn{1}{c}{ DISADVANTAGES } \\
\hline Feedback - twenty-three students (5I per cent) & $\begin{array}{l}\text { Problems with network and logins - eleven students } \\
\text { (24 per cent) } \\
\text { Lack of tutor support - four students (8 per cent) }\end{array}$ \\
$\begin{array}{l}\text { Easy access to resource - fourteen students } \\
\text { (3 per cent) }\end{array}$ & $\begin{array}{l}\text { Less keen if lack skills or desire to use computer - three } \\
\text { Individual use - fourteen students (31 per cent) }\end{array}$ \\
\hline
\end{tabular}

Figure 4: Perceived advantages and disadvantages of using the tool

Similarly, students were asked to list their favourite and least favourite aspects of the tool. The three most common responses are listed in Figure 5 and reflect the advantages and disadvantages. Again, 'problems with running' may be attributed to network performance on one day of the pilot. Four students were aggrieved that correct answers were not provided; this however, was a deliberate decision in order that students would engage more actively with the materials.

\begin{tabular}{ll}
\hline \multicolumn{1}{c}{ BEST } & \multicolumn{1}{c}{ LEAST } \\
\hline Feedback - twelve students (twenty-seven per cent) & $\begin{array}{l}\text { Problems with running - twelve students } \\
\text { (26 per cent) } \\
\text { Glossary - eleven students (twenty-four per cent) }\end{array}$ \\
$\begin{array}{l}\text { Videos not next to questions - eight students } \\
\text { (18 per cent) } \\
\text { Easy to use - four students (eight per cent) }\end{array}$ & $\begin{array}{l}\text { Correct answers not provided - four students } \\
\text { (eight per cent) }\end{array}$ \\
\hline
\end{tabular}

Figure 5: Fovourite and least fovourite aspects of the tool

Finally students were asked for ideas for improvement or any other comments. The most common suggestions and comments are summarized as: 
- Have correct answers provided - seven students (16 per cent)

- Group muscle choices - five students (11 per cent)

- Beneficial to assist in learning the subject and prepare for the exam - ten students (22 per cent)

- Good idea - seven students (16 per cent)

\section{Discussion}

As the study relates to the use of C\&IT for particular aspects of anatomy, it is difficult to generalize conclusions. However, a large corpus of research exists in the biomedical sciences which suggests that computer-based learning can be at least as effective as traditional independent study. It is evident that students tested during the pilot had a generally positive attitude towards the technology, a factor regarded as impacting significantly on successful uptake by Gunn and Maxwell (1996) in a similar study. This complements other research which suggests that physiotherapy students, occupational therapy students (Williams, Agho and Holm, 1996) and nursing students (Saranto, 1998; Cohen and Dacany, 1994) have a positive attitude to computer-based learning and desire to increase their knowledge and skills in this area. It will therefore be important to ensure that future use of the tool by students on other health courses is underpinned by sound training in basic C\&IT skills.

Comments revealed that students welcomed the tool as an aid to independent study, provided it was in addition to, and not supplanting, the tutor-supported practical sessions. Brain, Dewhurst and Williams (1999), conducting a similar evaluation for CBL tool in pharmacology, found similar attitudes amongst their pilot group. There may be a reluctant adoption of learning technologies in health-related courses as they are traditionally practice-based. Arguably, students may be concerned that a computer simulation of clinical practice will not adequately prepare them for real patient situations, and they prefer to trust the clinical experience of tutors. Nevertheless, such attitudes can change: Leathard and Dewhurst (1995) report that medical students found CBL an acceptable alternative to live demonstrations. Although Brudenell and Carpenter (1990) report negative attitudes to their CBL tool post-test, they suggest that one of the responsible factors could be that subject expectations were not met. In the current study the increased confidence evident amongst the pilot group after the test, suggests that these needs were met, as comments made by the students relate directly to the course objectives.

Clearly, although the positive results are encouraging, it is important to remember that the success of the tool (as with all learning technologies) will depend on its effective integration into the module, and an assurance that students will have access to help and support from the teaching team as they need it. As noted above, this tool is intended to be supportive, not supplantive, of tutor-led practical sessions.

\section{Future developments}

The Movement Analysis Tool will be extended during next year to include a full library of normal movements. If students continue to utilize the tool for their study, it is hoped in due course to add case studies of specially filmed dysfunctional movements which would 
integrate into higher levels of the course. This would enable the tool to become a progressive learning theme throughout the entire degree course. It would also allow students to practise their analysis of dysfunctional movement before they undertake their clinical placement.

The positive response to the tool from students during the pilot would seem to indicate that the tool did achieve the aims specified above. If an effective methodology can be devised it will be interesting to see how, if at all, the tool affects students' performance in exams.

The tool was demonstrated at two conferences last year - Alt-C 99 and CAL 99. A considerable amount of interest was generated, not just from other HE institutions, but also from sports colleges and other teaching practitioners. There is thus the potential for the tool to be shared nationally or made available commercially. This would give other clinicians the opportunity to contribute to the content and thus develop the tool on a national or regional basis.

\section{References}

Brain S., Dewhurst, D. G. and Williams, A. D. (1999), 'Evaluation of the usefulness of a computer-based learning programme to support student learning in pharmacology', $A L T$ $J, 7(2), 37-45$.

Brudenell, I. and Carpenter, C. S. (1990), 'Adult learning styles and attitudes toward computer assisted instruction', Journal of Nursing Education, 29 (2), 79-83.

Carr J. H., Shepherd R. B. and Ada L. (1995), 'Spasticity: research findings and implications for intervention', Physiotherapy, 81 (8), 421-9.

Cohen, P. A. and Dacanay, L. S. (1994), 'A meta-analysis of computer based instruction in nursing education', Computers in Nursing, 12 (2), 89-97.

French, S., Neville, S. and Laing, J. (1994), Teaching and Learning: A Guide for Therapists, Oxford: Butterworth-Heinemann.

Gunn, C. and Maxwell, L. (1996), 'CAL in human anatomy', Journal of Computer Assisted Learning, 12, 205-15.

Kielhofner, G. (1997), Conceptual Foundations of Occupational Therapy, 2nd edn, Philadelphia: F. A. Davis Company.

Leathard, H. L. and Dewhurst, D. G. (1995), 'Comparison of the cost-effectiveness of a computer-assisted learning program with a tutored demonstration to teach intestinal motility to medical students', $A L T-J, 3$ (1), 118-25.

Rothstein, J. M. (1986), 'Pathokinesiology - a name for our times?', Physical Therapy, 66 (3), 364-5.

Saranto, K. (1998), 'Outcomes of education in information technology at nursing polytechnics', Health Informatics Journal, 4 (2), 84-91.

Snell, R. S. (1995), Clinical Anatomy for Medical Students, 5th edn, New York: Littlebrown and Company. 
Watson, M. J. (1999), 'Clinical reasoning in neurology: Perry's model', Physiotherapy, 85 (5), 281-8.

Williams, A. M., Agho, A. O. and McCloy, C. (1996), 'Attitudes and perceptions of computer applications among baccalaureate physical therapy students', Journal of Physical Therapy Education, 10 (1), 14-17.

Williams, A. M., Agho, A. O. and Holm, M. B. (1996), 'Perceptions of computer literacy among occupational therapy students', American Journal of Occupational Therapy, 50 (3), 217-22.

Williams-Pedretti, L. and Zoltan, B. (1990), Occupational Therapy: Practice Skills for Physical Dysfunction, 3rd edn, Toronto: CV Mosby Company. 